\title{
Monitoring the profile variability of the double-peaked Balmer lines in Arp 102B
}

\author{
Suvi Gezari $^{1}$, J. P. Halpern ${ }^{1}$, M. Eracleous ${ }^{2}$ and A. V. Filippenko ${ }^{3}$ \\ ${ }^{1}$ Columbia University, New York, NY 10027, USA email: suvi@astro.columbia.edu \\ ${ }^{2}$ Pennsylvania State University, USA \\ ${ }^{3}$ University of California, Berkeley, USA
}

\begin{abstract}
We present the results of over 20 years of optical spectroscopic monitoring of the prototypical double-peaked emitter, Arp 102B. Its distinct double-peaked, extremely broad (FWHM $\approx 15,000 \mathrm{~km} \mathrm{~s}^{-1}$ ) low-ionization lines $(\mathrm{H} \alpha, \mathrm{H} \beta$, and $\mathrm{Mg} \mathrm{II})$ are well-modeled by emission from photoionized gas in a relativistic Keplerian accretion disk around a central black hole (Chen \& Halpern 1989; Halpern et al. 1996). A ubiquitous property of double-peaked emitters is longterm variability in the shape of their line profiles on the timescale of months to years, which is consistent with the dynamical timescale of an accretion disk. This variability can be attributed to inhomogeneities in the line-emitting disk, i.e., hot spots, spiral arms, eccentricity, and warps. We characterize the profile variability of Arp 102B in detail, and use it to constrain physical models for the structure and dynamics of its line-emitting accretion disk.
\end{abstract}

\section{Introduction}

Newman et al. (1997) studied the profile variability of the double-peaked $\mathrm{H} \alpha$ line in Arp 102B over 13 years, and found a sinusoidal variation of the red-to-blue peak flux ratio from 1990 to 1994, which they modeled as a transient orbiting hot spot in the accretion disk. A study by Sergeev et al. (2000) monitored the broad $\mathrm{H} \alpha$ line from 1992 to 1996 , and also found the variations in the profile to be suggestive of gas rotating in a disk with inhomogeneities in the surface brightness, but favored the geometry of a disk composed of orbiting discrete clouds. We extend the baseline of these studies to the present, with spectra obtained from 1983 to 2004 with 2-meter-class telescopes (including the MDM $2.4 \mathrm{~m}$, KPNO $2.1 \mathrm{~m}$, and Lick $3 \mathrm{~m}$ ), in order to further test and improve the proposed models for the source of the double-peaked Balmer lines and their variability.

\section{Trends in Long-Term Variability: Constraining Physical Models}

A viable model for the structure and dynamics of the line-emitting gas must be able to reproduce the following trends we measure in the long-term variability of the doublepeaked $\mathrm{H} \alpha$ and $\mathrm{H} \beta$ profile shape (shown in Figure 1):

- Transient periodic oscillation of the red-to-blue peak flux ratio.

- Drop in red-to-blue peak flux ratio, peak separation, and full width at quarter maximum (FWQM) during flares in line flux and continuum.

- An excess in the blue peak during flares, in comparison to the low-state mean profile.

- A systematic drift in the red peak velocity (with possible periodic behavior), with a stationary blue peak velocity.

- A trend towards smaller peak separation and FWQM over time, with possible periodic behavior.

The orbiting binary broad-line region model, an alternative to the accretion disk model, is strongly rejected by our results, due to the stability of the velocity of the blue peak 

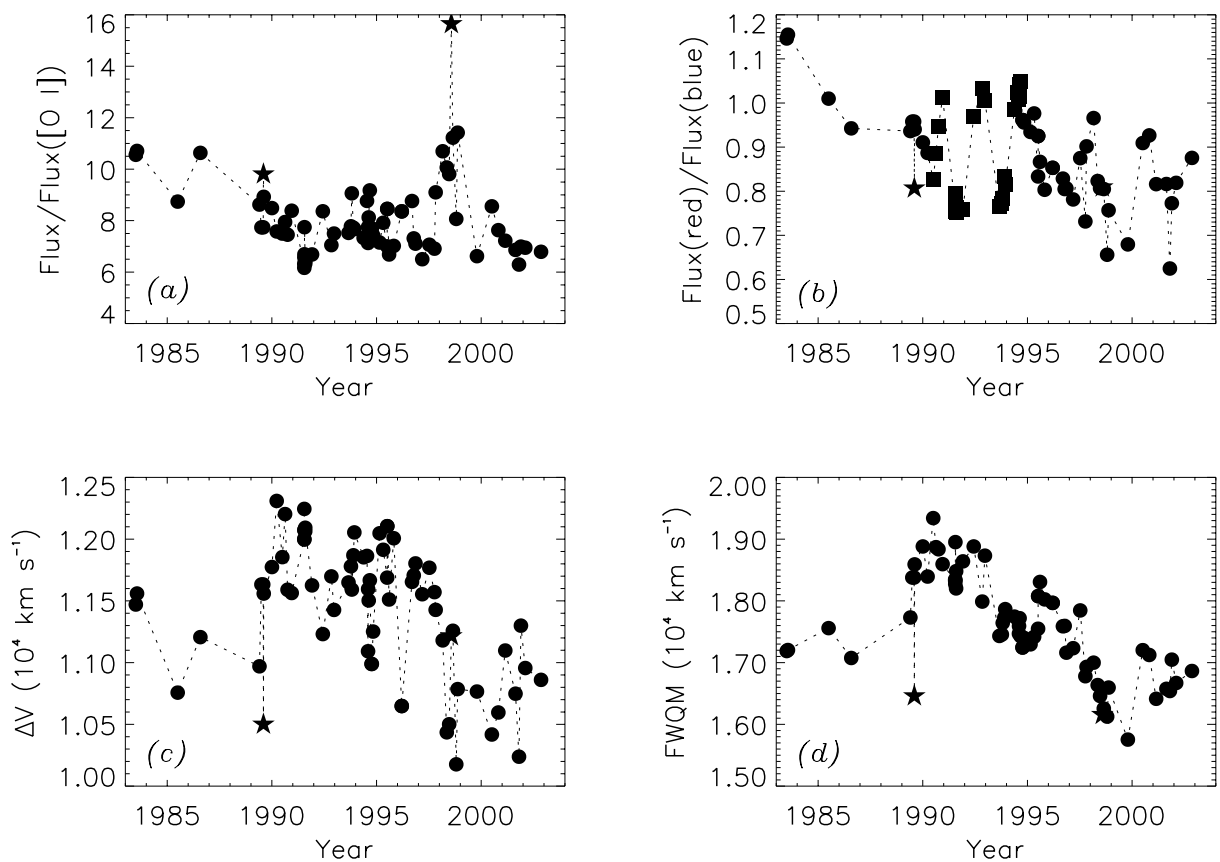

Figure 1. Characterization of the broad double-peaked $\mathrm{H} \alpha$ profile variability. (a) Flux of broad $\mathrm{H} \alpha$, (b) ratio of flux in red peak to flux in blue peak, (c) velocity separation of peaks, and (d) FWQM over time. Times of flares in flux are plotted with stars, and previously suggested periodic variability is plotted with squares.

for over 20 years. The disk model can account for both the periodic oscillation of the red-to-blue peak flux ratio, and the decreased separation between peaks during flares in flux, if one imposes an inhomogeneity in the emissivity of the disk, and if the flare in ionizing continuum is illuminating lower velocity material in the outer parts of the disk. What must be studied further is if a disk is consistent with the excess in the blue peak during flares, and what could cause the drift of the velocity of the red peak, with the blue peak remaining relatively stationary, as well as long-term, systematic changes in the peak separation and FWQM of the profile. Progress in modeling the structure of the broad-line gas in double-peaked emitters is critical for furthering our general understanding of the nature of the broad-line region in active galactic nuclei.

\section{Acknowledgements}

Travel to the conference by was supported in part by the NSF International Travel Grant, administered by the AAS. AVF is grateful for NSF grant AST-0307894.

\section{References}

Chen, K., \& Halpern, J. 1989, ApJ, 344, 115

Eracleous, M., Halpern, J., Gilbert, A., Newman, J. A., \& Filippenko, A.V. 1997, ApJ, 490, 216

Halpern, J. P., Eracleous, M., Filippenko, A. V., \& Chen, K. 1996, ApJ, 464, 704

Newman, J. A., Eracleous, M., Filippenko, A. V., \& Halpern, J. 1997, ApJ, 485, 570

Sergeev, S. G., Pronik, V. I., \& Sergeeva, E. A. 2000, A\&A, 356, 41 